\title{
Three-dimensional macroporous silicon photonic crystal with large photonic band gap
}

\author{
J. Schilling, ${ }^{\text {a) }}$ J. White, and A. Scherer \\ California Institute of Technology, 391 S. Holliston Avenue, Pasadena, California 91125
}

G. Stupian

The Aerospace Corporation, Los Angeles, California

R. Hillebrand and U. Gösele

Max-Planck Institute of Microstructure Physics, Halle, Germany

(Received 30 April 2004; accepted 25 October 2004; published online 22 December 2004)

\begin{abstract}
Three-dimensional photonic crystals based on macroporous silicon are fabricated by photoelectrochemical etching and subsequent focused-ion-beam drilling. Reflection measurements show a high reflection in the range of the stopgap and indicate the spectral position of the complete photonic band gap. The onset of diffraction which might influence the measurement is discussed. () 2005 American Institute of Physics. [DOI: 10.1063/1.1842855]
\end{abstract}

Three-dimensional (3D) photonic crystals with 3D complete photonic band gaps exhibit interesting optical properties. First they promise to allow the inhibition of spontaneous emission ${ }^{1-4}$ for light frequencies within the gap. Moreover, the 3D confinement of light at defects eliminates scattering losses and can therefore lead to high $Q$ microresonators and efficient photonic crystal waveguides. Indeed 3D photonic crystals have been fabricated and are known as Yablonovites, ${ }^{5,6}$ Lincoln log structures, ${ }^{7,8}$ or self-ordered inverse opals. ${ }^{9}$ However, the fabrication of structures with band gaps in the near infrared or visible spectral region is difficult (Yablonovite), tedious (Lincoln $\log$ ), or the structures suffer from disorder (self-ordered opals). This limits the sizes of perfect structures to a few lattice constants. To achieve a more extended Yablonovite-like 3D photonic crystal Lourtioz et al. ${ }^{10,11}$ used a combination of photoelectrochemical macropore etching in silicon and subsequent drilling of two pore sets with a focused ion beam (FIB). The FIB drilling of the etched macroporous silicon is faster than of bulk material and the problem of redeposition of milled material is minimized at the same time. We apply this technique to fabricate an alternative 3D photonic crystal structure recently proposed by Hillebrand et al. ${ }^{12}$ which consists only of two orthogonal interpenetrating pore sets in a high index material (Fig. 1). Each pore set of our structure consists of a pattern of two-dimensional (2D) hexagonally arranged pores forming a 3D structure of orthorhombic symmetry with the

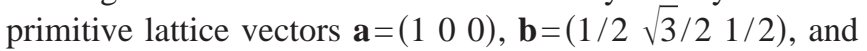
$\mathbf{c}=\left(\begin{array}{lll}0 & 0 & 1\end{array}\right)$.

The resulting first Brillouin zone resembles the slightly distorted Brillouin zone of a fcc lattice (Fig. 2(a)). We performed band-structure calculations using silicon with a refractive index of 3.5 as the matrix material applying the MPB program developed by Johnson. ${ }^{13}$ When the ratio of the pore radius $r$ to lattice constant $a$ of both pore sets is $r / a=0.38$, a maximum complete 3D photonic band gap of $\Delta \omega / \omega_{\text {center }}$ $=25.1 \%$ is obtained (Fig. 2(b)). The gap appears at low normalized frequencies in the band structure between the second and third bands and should therefore be insensitive to modest disorder.

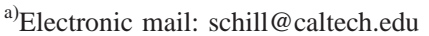

We realized the described structure by first creating a 2D hexagonal pattern of etch pits applying a $\mathrm{KOH}$ solution on the (100) surface of an $n$-type silicon wafer. The position of the etch pits and the lattice constant of $a=500 \mathrm{~nm}$ were lithographically defined. Afterwards the structured side of the wafer was immersed in hydrofluoric acid and a photoelectrochemical etch process ${ }^{14}$ was used to create 50 - $\mu$ m-deep macropores in the silicon wafer starting at the etch pits. The radius of the photoelectrochemically etched pores $r_{\text {etch }}$ $=190 \mathrm{~nm}$ was determined by the etch current. This pore pattern already forms a 2D photonic crystal ${ }^{15-17}$ and the characteristic 2D band gaps were observed in reflection measurements confirming the given structure parameters $a$ and $r_{\text {etch }}$. The structure was then cleaved and a 100- $\mu$ m-long trench was milled out of the porous region applying a dual beam FIB (model DB235 from FEI). The trench was positioned $20 \mu \mathrm{m}$ away from the cleaved facet so that a $100-\mu \mathrm{m}$-long and $20-\mu \mathrm{m}$-wide ridge of porous material was clearly separated from the rest of the sample. Now the second pore set was drilled with the FIB from the side into the cleaved facet. The direction of the ion beam was perpendicular to the cleaved facet and therefore orthogonal to the first pore set. Applying an ion current of $420 \mathrm{pA}$ a single pore penetrating the $20-\mu \mathrm{m}$-wide ridge could be drilled in 5 s. 275 pores, which were again arranged in a 2D hexagonal lattice with lattice constant of $500 \mathrm{~nm}$, were drilled successively within a $10-\mu \mathrm{m}$-long and 5- $\mu \mathrm{m}$-high field. To facilitate optical measurements, ten of these fields were stitched together laterally to form a total drilled volume of $100 \mu \mathrm{m} \times 5 \mu \mathrm{m} \times 20 \mu \mathrm{m}$.

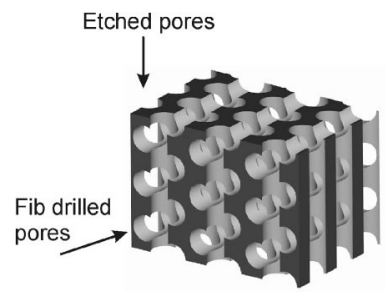

(a)

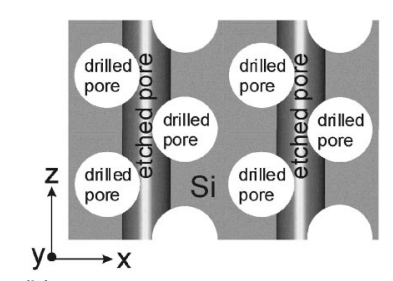

(b)
FIG. 1. Model of the orthorhombic structure consisting of two hexagonal pore sets: (a) 3D view revealing the interpenetrating network of pores, (b) side view along the FIB-drilled pores showing the relative position of etched and drilled pores. 


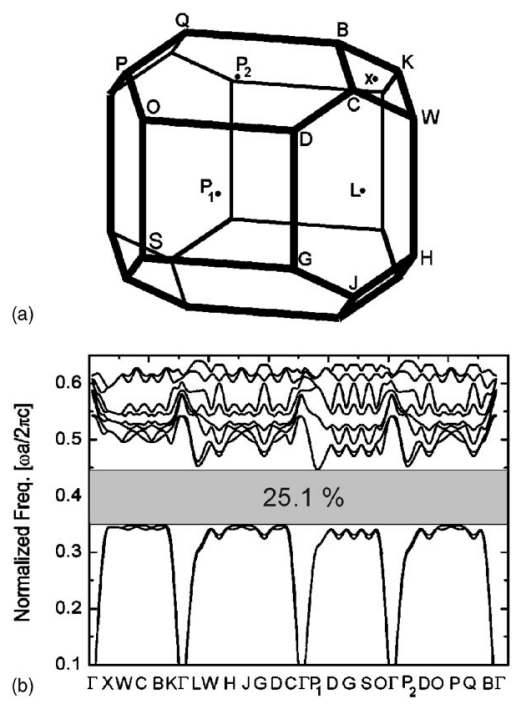

FIG. 2. (a) First Brillouin zone for the orthorhombic structure. (b) Band structure for the orthorhombic structure with $r / a=0.38$ for both pore sets.

Figure 3(a) shows a tilted overview of the structure. In front the cleaved facet with the perpendicularly running photoelectrochemically etched pores is visible. In the upper half of the picture the surface of the sample including the trench is shown. The drilled pores are only situated in the upper $5 \mu \mathrm{m}$ of the ridge directly below the surface. A closer look to the far side of the trench reveals the drilled hexagonal pore pattern even there. It was created by impinging ions which penetrated the ridge and crossed the trench before. This is a clear proof that the FIB drilled pores penetrate the whole ridge and that the drilled pore pattern stays intact over the whole drilling depth.

To obtain the large $10 \mu \mathrm{m} \times 5 \mu \mathrm{m}$ drilled fields and to reduce therewith the number of stitching interfaces, the magnification of the FIB image was reduced. Although this complicates the correct placement of the drilled pores with respect to the etched pores, a close inspection of the drilled pores with the SEM reveals that they are only shifted by

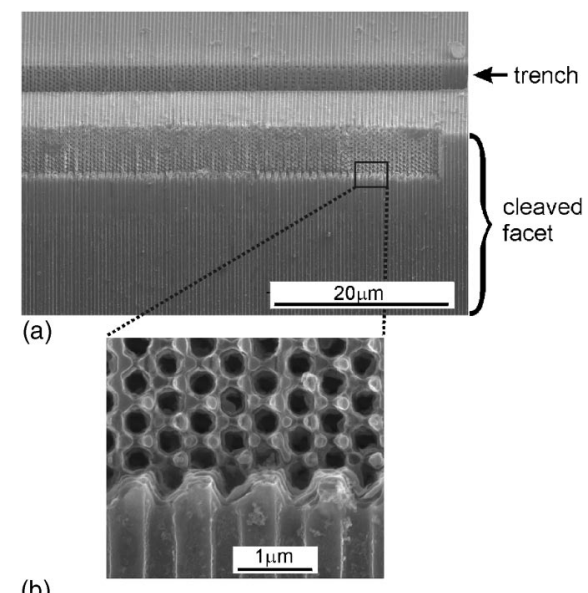

(b)

FIG. 3. SEM image of the prepared sample. (a) Tilted overview. In front the perpendicular running etched pores are visible. In their upper $5 \mu \mathrm{m}$ the orthogonal pore set was drilled with the FIB. Due to the tilt the milled trench is visible too. It separates the ridge containing the drilled structure from the rest of the sample. (b) Detailed view of the the drilled region showing the alignment of etched pores (perpendicular running cut pores at the bottom) and drilled pores (visible as hexagonal hole pattern). The periodicity of the drilled pores perfectly matches the lattice constant of the etched pores. The

drilled pores run in between the etched pores as intended. region of the drilled sample.
Downloaded 14 Dec 2005 to 131.215 .225 .171 . Redistribution subject to AlP license or copyright

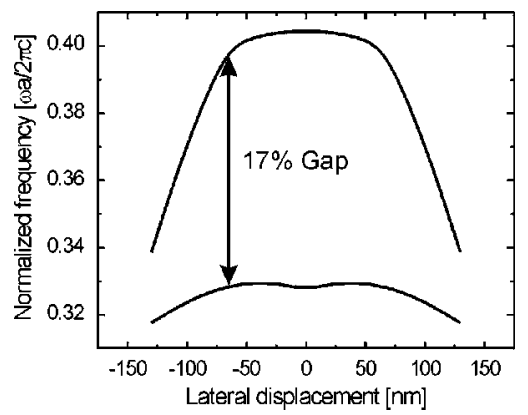

FIG. 4. Position of the lower and upper band edges depending on the displacement of the drilled pore set with respect to the etched pores. The lattice constant is $a=500 \mathrm{~nm}$, the radius of the etched pores is $r_{\text {etch }}=190 \mathrm{~nm}$, and the radius of the drilled pores is $r_{\text {drill }}=175 \mathrm{~nm}$.

$-65 \mathrm{~nm}$ from their ideal positions (Fig. 3(b)). The radius of the drilled pores is observed to be $r_{\text {drill }}=175 \mathrm{~nm}$. To investigate how these slight deviations of the fabricated structure influence the band gap, band-structure calculations considering $r_{\text {etch }}=190 \mathrm{~nm}$ and $r_{\text {drill }}=175 \mathrm{~nm}$ were performed and the displacement of the drilled pore set with respect to the etched pore set was varied. Figure 4 shows the position of the lower (dielectric) and the upper (air) band edge for the different values of the displacement. A displacement of 0 describes the ideal structure, where the drilled pores run exactly in between the etched pores. These calculations show that our fabricated structure, which has a $-65 \mathrm{~nm}$ displacement, should still exhibit a complete gap of $17 \%$.

To test the optical properties of our structures, we carried out reflection measurements along the $z$ direction. An infrared microscope, which was attached to a Fourier transform infrared (FTIR) spectrometer was used to focus the infrared light to an area of $30 \mu \mathrm{m} \times 20 \mu \mathrm{m}$ at the top surface. The mirror objective had a numerical aperture of n.a. $=0.57$, which corresponds to a cone of incident rays with $35^{\circ}$ opening angle. Unpolarized radiation was used. The reflection of a gold mirror was taken as a reference and considered to be unity.

The reflection of the drilled sample is shown in Fig. 5. A spectral region of high reflectivity between $6000 \mathrm{~cm}^{-1}(1.66 \mu \mathrm{m})$ and $8000 \mathrm{~cm}^{-1}(1.25 \mu \mathrm{m})$ can be clearly observed. This agrees well with the predicted position of the stop gap $\left(6100-8080 \mathrm{~cm}^{-1}\right)$ along the $z$ direction in

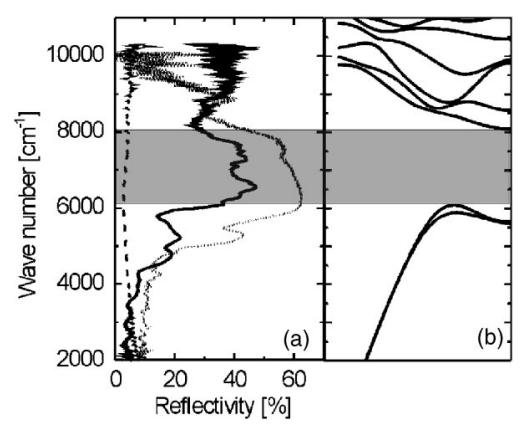

FIG. 5. (a) Unpolarized reflection measurement of the drilled sample (solid), an undrilled region parallel to the etched pores (dashed) and an undrilled region perpendicular to the etched pores ( $\Gamma-K$ direction) (dotted). A spectral region of high reflectivity appears in the spectrum of the drilled sample between 6000 and $8000 \mathrm{~cm}^{-1}$. A similar high reflectivity is observed for the undrilled sample with an incidence perpendicular to the etched pore set. (b) Section of the band structure along the investigated $z$ direction. The stopgap (indicated by the grey bar) overlaps very well with the high reflectivity region of the drilled sample. 
the corresponding section of the band structure. This consistency indicates that the fabricated structure really corresponds to the model used for the band structure calculations. For comparison, the reflection parallel and perpendicular to the etched pores of an undrilled part of the sample is shown in Fig. 5(a). The reflectivity parallel to the pore axis is much lower and does not show any significant features as no periodic structure exists in this propagation direction. In contrast the reflection perpendicular to the pores, where the light is incident on the cleaved side of the undrilled sample, is strongly increased between $5000^{-1}$ and $8000 \mathrm{~cm}^{-1}$ caused by the periodic arrangement of the pores in the plane of propagation. This corresponds to the reflection of a macroporous silicon 2D photonic crystal exhibiting a band gap and noncoupling bands along the $\Gamma-K$ direction. ${ }^{17} \mathrm{~A}$ comparison of this reflection from the side of the undrilled region (2D crystal) with the reflection from the top of the drilled region (3D crystal) shows a strong similarity. Indeed the periodicity along the respective propagation directions is alike. In both cases a hexagonal pore set, which is oriented perpendicular to the propagation direction $(\Gamma-K$ direction of the $2 \mathrm{D}$ hexagonal pore sets), exists. For the undrilled 2D crystal this is the etched pore set while for the drilled 3D crystal it is the FIB-drilled pore set. The absence of any features in the reflection parallel to the undrilled pores and the similarity of the high reflections of the 3D crystal from the top and the 2D crystal from the side prove experimentally that the strong reflection of the drilled 3D crystal is really caused by the introduction of the FIB-drilled hexagonal pore set.

As a photonic crystal is a periodic medium, the whole backreflected light from the crystal in general comprises specular reflection (zero-order diffraction) and diffraction into higher orders. Depending on the exit angle these diffracted rays might not enter the objective lens of the microscope leading to a reduced detected overall backreflection. These "diffraction losses" can be the reason for a detected reflectivity below $100 \%$ in the spectral region of a photonic band gap. However for low frequencies $(\omega \rightarrow 0)$ only specular reflection exists. The first diffraction order can only appear when the component of the diffracted wave vector which is parallel to the surface of the crystal just equals the wave vector in air $\left(\mathbf{k}_{\text {air }}\right)$ for this frequency. The parallel component of the diffracted wave vector is the sum of the parallel component of the incident wave vector $\left(k_{\|}^{\text {in }}\right)$ and the parallel component $\left(G_{\|}\right)$of any reciprocal lattice vector $\mathbf{G}$. These necessary conditions can be cast in the form

$$
k_{\|}^{\text {in }} \pm G_{\|} \leqslant\left|\mathbf{k}_{\text {air }}\right| .
$$

To determine the lowest frequency for which diffraction of our structure can appear, only those reciprocal lattice vectors with the smallest nonzero parallel components have to be considered. In our structure this is $G_{\|}=1 / \sqrt{3} a$. For strict normal incidence $\left(k_{\|}^{\text {in }}=0\right)$ condition (1) yields $\left|\mathbf{k}_{\text {air }}\right| \geqslant 1 / \sqrt{3} a$ $=11600 \mathrm{~cm}^{-1}$ where the lattice constant $a=500 \mathrm{~nm}$ was inserted. This is above the frequency range of our experiment. If we had only strict normal incidence in our experiment, diffraction would not occur in the investigated frequency range and the reflectivity in the gap region should ideally reach unity. However a mirror objective with n.a. $=0.57$ was used for the reflection measurement, so that the most oblique ray had an angle of incidence of $35^{\circ}$. For this maximum angle Eq. (1) yields $\left|\mathbf{k}_{\text {air }}\right| \geqslant \sin 35^{\circ} \cdot\left|\mathbf{k}_{\text {air }}\right|-1 / \sqrt{3} a$
$=7340 \mathrm{~cm}^{-1}$. Diffraction therefore appears for some of the off-normal rays at frequencies above $7340 \mathrm{~cm}^{-1}$. The diffracted light at these relatively low frequencies exits the sample surface at large angles and is not collected by the objective. The measured reflectivity is therefore diminished and cannot reach unity in the range $7340-8080 \mathrm{~cm}^{-1}$ although a photonic stopgap is present in this spectral region. In the range from 6100 to $7340 \mathrm{~cm}^{-1}$ however the reflectivity should ideally still reach unity as the band gap is present and no diffraction occurs so that incident light is only specularly reflected. Probably a slight surface roughness or the roughness at the pore walls leads to the higher scattering losses limiting the maximum observed reflectivity to $50 \%$ within the gap.

In conclusion, an orthorhombic 3D photonic crystal consisting of two interpenetrating hexagonal pore sets was fabricated on the basis of macroporous silicon applying a photoelectrochemical etch process and FIB drilling. Reflection measurements reveal a stopgap along the direction of incidence which corresponds very well with the band structure of the model. This agreement indicates that the fabricated structure corresponds to the theoretical model structure and is therefore a very promising candidate to observe a $3 \mathrm{D}$ complete photonic band gap experimentally in the future. To prove a 3D complete photonic band gap unambiguously, time-resolved measurements of emitting substances which are placed in the 3D crystal have to be performed. A strong extension of lifetime would be the signature of the inhibition of spontaneous emission and is considered to be the experimental proof of a complete 3D photonic band gap.

J.S. and J.W. would like to thank George R. Rossman for providing the opportunity to use the FTIR spectrometer and microscope for the reflection measurements.

${ }^{1}$ E. Yablonovitch, Phys. Rev. Lett. 58, 2059 (1987).

${ }^{2}$ S. John and J. Wang, Phys. Rev. B 43, 12772 (1991).

${ }^{3}$ T. Quang, M. Woldeyohannes, S. John, and G. S. Agarwal, Phys. Rev. Lett. 79, 5238 (1997).

${ }^{4}$ S. John and M. Florescu, J. Opt. A, Pure Appl. Opt. 3,S103 (2001).

${ }^{5}$ E. Yablonovitch, T. J. Gmitter, and K. M. Leung, Phys. Rev. Lett. 67, 2295 (1991).

${ }^{6}$ C. C. Cheng and A. Scherer, J. Vac. Sci. Technol. B 13, 2696 (1995).

${ }^{7}$ S. Y. Lin, J. G. Fleming, D. L. Hetherington, B. K. Smith, R. Biswas, K. M. Ho, M. M. Sigalas, W. Zubrzycki, S. R. Kurtz, and J. Bur, Nature (London) 394, 251 (1998).

${ }^{8}$ S. Noda, K. Tomoda, N. Yamamoto, and A. Chutinan, Science 289, 604 (2000).

${ }^{9}$ A. Blanco, E. Chomski, S. Grabtchak, M. Ibisate, S. John, S. W. Leonard, C. Lopez, F. Meseguer, H. Miguez, J. P. Mondia, G. A. Ozin, O. Toader, and H. M. Van Driel, Nature (London) 405, 437 (2000).

${ }^{10}$ A. Chelnokov, K. Wang, S. Rowson, P. Garoche, and J.-M. Lourtioz, Appl. Phys. Lett. 77, 2943 (2000).

${ }^{11}$ K. Wang, A. Chelnokov, S. Rowson, and J.-M. Lourtioz, Appl. Phys. A: Mater. Sci. Process. 76, 1013 (2003).

${ }^{12}$ R. Hillebrand, S. Senz, W. Hergert, and U. Gösele, J. Appl. Phys. 94, 2758 (2003).

${ }^{13}$ S. G. Johnson, http://ab-initio.mit.edu/mpb/, MIT.

${ }^{14}$ V. Lehmann, J. Electrochem. Soc. 140, 2836 (1993).

${ }^{15}$ A. Birner, U. Grüning, S. Ottow, A. Schneider, F. Müller, V. Lehmann, H. Föll, and U. Gösele, Phys. Status Solidi A 165, 111 (1998).

${ }^{16}$ J. Schilling, R. B. Wehrspohn, A. Birner, F. Müller, R. Hillebrand, U. Gösele, S. W. Leonard, J. P. Mondia, F. Genereux, H. M. van Driel, P. Kramper, V. Sandoghdar, and K. Busch, J. Opt. A, Pure Appl. Opt. 3, S121-S132 (2001).

${ }^{17}$ S. Rowson, A. Chelnokov, C. Cuisin, and J.-M. Lourtioz, J. Opt. A, Pure Appl. Opt. 1, 483 (1999). 